\title{
O USO DO CUSTEIO BASEADO EM ATIVIDADES - ABC (ACTIVITY BASED COSTING) NAS MAIORES EMPRESAS DE SANTA CATARINA
}

\author{
Ilse Maria Beuren \\ Professora Titular do Departamento de Ciências Contábeis da Universidade Federal de Santa Catarina \\ E-mail: beuren@cse.com.br
}

\section{Ari Roedel}

Mestre em Administração: Gestão Moderna de Negócios da Universidade Regional de Blumenau - FURB E-mail: beuren@cse.com.br

RESUMO

Devido à globalização da economia, as empresas evoluíram para a produção de enormes variedades de produtos personalizados. Com o intuito de atender às exigências dos clientes, surgiu a necessidade de grandes alterações nos processos tecnológicos. Como conseqüência, os custos indiretos passaram a ser mais significativos, o que implicou um novo método de distribuição desses custos, capaz de distribuir melhor os custos indiretos aos produtos e serviços. A esse método foi dado o nome de custeio baseado em atividades - ABC (Activity Based Costing). Esse método foi investigado por Carlos Yorghi Khoury em 1996, numa pesquisa aplicada às 500 maiores empresas do Brasil. O objetivo deste trabalho foi replicar essa pesquisa nas maiores empresas catarinenses, a fim de observar se esses resultados obtidos assemelham-se aos obtidos na pesquisa com as empresas catarinenses. Nesta pesquisa, dos 200 questionários enviados para as maiores empresas de Santa Catarina, 81 retornaram com as questões respondidas. O trabalho levou a conclusões importantes, como: as empresas de Santa Catarina têm amplo conhecimento do sistema $\mathrm{ABC}$; algumas empresas que implantaram esse sistema já estão desistindo de seu uso, em função da complexidade para apropriar os custos indiretos e as despesas às atividades e estas aos produtos.

Palavras-chave: custeio baseado em atividades, recursos, atividade, produtos.
ABSTRACT

Due to the economic globalization, enterprises have started to produce enormous varieties of personalized products. In order to attend to the requirements of their clients, huge changes in the technological process were needed. Consequently, indirect costs became more significant, which led to a new method for distributing the indirect costs, capable of better distributing the indirect costs of products and services. This method was called Activity Based Costing and was investigated by Carlos Yorghi Khoury in 1996 through a research applied in the 500 biggest companies in Brazil. This paper aims to replicate the 1996 research in the biggest companies from Santa Catarina, Brazil, in order to observe if the results obtained from these companies are similar to those from the 1996 research. For the empirical research, 81 out of 200 questionnaires sent to the biggest companies from Santa Catarina were duly returned. The research led to the conclusion that, although the companies from Santa Catarina have a large knowledge of the $A B C$ system, some of the companies that introduced this system are already giving it up in function of the complexity in appropriating the cost and the indirect expense from the activities to the products.

Key words: activity based costing, resources, activities, products. 


\section{CONSIDERAÇÕES INICIAIS}

As últimas décadas têm sido marcadas por profundas mudanças no mundo dos negócios. Devido à globalização da economia, as empresas passaram a focar seus esforços no aumento da produtividade com redução de custos, o que só é possível com um sistema de informações flexível e ágil, que forneça informações para a administração acompanhar a dinâmica do mercado.

Os sistemas tradicionais, conhecidos como sistemas de custeio baseados em volumes, foram desenhados para empresas que competiam no mercado com base em estratégicas de redução de custos de produtos homogêneos e manufaturados em grande escala para estoque. Estes sistemas apropriavam os custos indiretos com base em alguns atributos diretamente relacionados com o volume de produção, tais como horas de mão-de-obra direta, horas-máquinas, valor de material consumido e outros critérios.

A nova abordagem requerida na administração dos custos, voltada à máxima produtividade e à necessidade de maior controle sobre os custos indiretos, que hoje são muito mais significativos, fez surgir uma outra metodologia de apuração de custos. É o que tem sido utilizado com sucesso em muitas empresas industriais e de serviços, por tratar como base de custeio as atividades desempenhadas dentro das empresas, facilitando consideravelmente a análise do negócio por processos e eliminando a arbitrariedade dos critérios de rateio. Essa metodologia é denominada Custeio Baseado em Atividade - ABC (Activity Based Costing). O sistema de custeio baseado em atividades não se diferencia do sistema de custeio baseado em volume apenas pela mudança das bases de alocação de custos indiretos, mas também pela identificação que faz dos custos por atividade e pela maneira como aloca os custos aos produtos, através de maior número de bases.

De acordo com Brimson (1996:27), "a abordagem da contabilidade por atividades para gerenciamento de custos divide uma empresa em atividades. Uma atividade descreve o que uma empresa faz - a forma como o tempo é gasto e os produtos do processo. A principal função de uma atividade é converter recursos (material, mão-de-obra e tecnologia) em produtos e serviços. A contabilidade por atividades identifica as atividades desenvolvidas em uma empresa e determina seu custo e desempenho (tempo e qualidade)."

Chalos (1992:62) explicita que "a premissa básica do $A B C$ é que atividades consomem recursos e produtos consomem atividades. Isto significa que custos nos negócios devam ser primeiro acumulados no nível de atividades e de lá então para determinado produto. Os custos acumulados sob uma atividade são determinados para o produto usando direcionador de custo".

$O A B C$, que neste cenário vem se tornando cada vez mais popular, não é, entretanto, um sistema de acumulação de custos para fins contábeis, em substituição aos já existentes. É um sistema de custeio baseado na análise das atividades significativas desenvolvidas na empresa, com vistas à atribuição mais criteriosa dos gastos indiretos, seu controle mais efetivo e um meIhor suporte ao processo decisório.

\section{A PESQUISA}

Apesar de o sistema de custos $A B C$ ter sofrido constantes refinamentos nestes últimos anos, as controvérsias sobre sua utilização continuam. Várias pesquisas, conforme publicado por Carlos Yorghi Khoury na RAE - Revista de Administração de Empresas, da Fundação Getúlio Vargas, (1999:55-63), foram realizadas e publicadas nos Estados Unidos, Inglaterra, Canadá, Brasil, entre outros, sobre a utilização do ABC pelas empresas, as quais mostraram que as empresas na Inglaterra são as que mais têm aceito e adotado esse método, seguidas das empresas nos Estados Unidos. No Brasil, a pesquisa foi por ele realizada em 1996 nas 500 maiores empresas relacionadas na Revista Exame - Melhores e Maiores, a qual tem mostrado que o número de empresas que estão implantando e/ou utilizando o sistema $A B C$ é muito pequeno, representando apenas 18\% (dezoito por cento) de uma amostra de 283 empresas que responderam ao questionário da pesquisa.

No entanto, a pergunta que fica é se no Brasil há diferenças, nos diversos Estados, no que concerne à extensão do conhecimento do sistema $A B C$ e como este está sendo aceito e implantado 
nas empresas. Nesta perspectiva, o objetivo deste trabalho foi replicar essa pesquisa nas maiores empresas catarinenses, a fim de verificar se os resultados alcançados por Carlos Yorghi Khoury assemelham-se aos dessa pesquisa.

Em Santa Catarina, a pesquisa foi realizada nas 200 maiores empresas, classificadas por número de empregados, relação conseguida junto ao Serviço de Informações Empresariais da Federação das Indústrias do Estado de Santa Catarina - FIESC. Do total de 200 questionários enviados, 81 empresas responderam às questões, representando $41 \%$ do montante dos enviados, cuja amostra é considerada representativa. Destacaram-se no setor de atuação as indústrias com $95 \%$; seguindo-se o comércio, com $4 \%$; e serviços, com $1 \%$ dos questionários respondidos. Em termos de tamanho, $47 \%$ das empresas possuem entre 500 e 2000 empregados. Do total, $82 \%$ possuem até 2000 empregados e $2 \%$ acima de 10.000 empregados.

\section{DESCRIÇÃO E ANÁLISE DOS DADOS COLETADOS}

Os dados da pesquisa foram tabulados e analisados enfocando a experiência das empresas em relação à utilização do $\mathrm{ABC}$, grau de acurácia do sistema de custos usado pelas empresas catarinenses, nível de conhecimento sobre o $A B C$ nas empresas de Santa Catarina, motivos que levaram as empresas catarinenses a implantar o $A B C$, razão do abandono do $A B C$ após implantado por empresas catarinenses e ligação do sistema $A B C$ a outros projetos de suporte à gestão das empresas catarinenses.

\subsection{Experiência das empresas em relação à utilização do ABC}

As empresas pesquisadas foram agrupadas de acordo com a experiência que tiveram em relação ao sistema ABC conforme mostra a Tabela 1.

\section{TABELA 1 - EMPRESAS COM EXPERIÊNCIA EM RELAÇÃO AO SISTEMA ABC}

\begin{tabular}{|lcr|}
\hline Experiência em relação ao sistema ABC & Noempresas & $\%$ \\
Empresas que não tinham ouvido falar do sistema ABC & 6 & 7,00 \\
\hline Empresas que tinham ouvido falar do sistema ABC, mas não se interessaram em adotá-lo & 47 & 58,00 \\
\hline Empresas que estavam avaliando o sistema ABC & 4 & 12 \\
\hline Empresas que tinham avaliado o sistema ABC e decidiram não utilizá-lo & 4 & 15,00 \\
\hline Empresas que tinham avaliado o sistema ABC e decidiram utilizá-lo & 1 & 5,00 \\
\hline Empresas que estavam implantando o sistema ABC & 5 & 1,00 \\
\hline Empresas que concluíram a implantação do sistema ABC e estavam utilizando-o & 6,00 \\
\hline Empresas que já utilizaram o sistema ABC e decidiram abandoná-lo & 2 & 2,00 \\
\hline Total & 81 & 100,00 \\
\hline
\end{tabular}

De um universo de 81 empresas pesquisadas, apenas 6 empresas, ou seja, 7\%, informaram que não tinham ouvido falar do sistema $A B C$. O setor da indústria foi o que representou esse percentual de empresas. Portanto, os dados indicam um alto índice de conhecimento desse sistema nas empresas de Santa Catarina que foram pesquisadas.
Observou-se que 58\%, isto é, aproximadamente dois terços das empresas tinham ouvido falar do sistema $A B C$, mas não se interessaram em adotá-lo. Perguntou-se a essas empresas sobre as razões pelas quais não se interessaram em adotar esse sistema, cujas respostas constam na Tabela 2. 
TABELA 2-RAZÕES PELAS QUAIS AS EMPRESAS NÃO SE INTERESSARAM EM UTILIZAR O SISTEMA ABC

\begin{tabular}{|lcc|}
\hline Razões do não interesse pelo sistema ABC & No Razões & \% \\
O sistema utilizado atende as necessidades da empresa & 14 & 30,00 \\
\hline Complexidade para a implantação e operação do sistema ABC & 9 & 19,00 \\
\hline O sistema ABC não é adequado para o seu negócio & 5 & 11,00 \\
\hline Falta de profundo conhecimento do sistema ABC & 4 & 9,00 \\
\hline Não quis responder & 4 & 9,00 \\
\hline Possibilidade de ele vir a ser implantado no futuro, como gerenciamento & 2 & 4,00 \\
\hline Alto custo e benefícios duvidosos & 2 & 4,00 \\
\hline Os custos indiretos não são representativos & 2 & 2,00 \\
\hline A utilização do sistema ABC não é prioritário & 1 & 2,00 \\
\hline A empresa possui um sistema semelhante ao sistema ABC & 1 & 2,00 \\
\hline Trabalhoso e raramente usado em empresas & 1 \\
\hline № elevado de informações, o custo p/mensuração sobrepõe os benefícios & 1 \\
\hline O ABC leva a resultados incertos pela subjetividade dos rateios & 1 \\
\hline Total & 47 & 2,00 \\
\hline
\end{tabular}

As respostas ressaltam de imediato dois aspectos. Primeiro, o valor do $A B C$ não se sobrepõe aos benefícios que a empresa já conhece no sistema que utiliza; segundo, observa-se que o sistema $A B C$ não empolga em função de sua complexidade.

As características motivadoras para implantação do sistema $A B C$ percebidas nas empresas catarinenses apresentam-se na Tabela 3.

\section{TABELA 3 - CARACTERÍSTICAS MOTIVADORAS PARA IMPLANTAÇÃO DO SISTEMA ABC}

\begin{tabular}{|c|c|}
\hline Características motivadoras para implantação do sistema $A B C$ & $\%$ \\
\hline $\begin{array}{l}\text { Análise do lucro mais acurada para tomada de decisões sobre que } \\
\text { produto produzir ou sobre que serviços fornecer }\end{array}$ & 57,00 \\
\hline $\begin{array}{l}\text { Discernimento das causas do aumento ou redução dos custos, } \\
\text { incluindo a habilidade de identificar os direcionadores de custos }\end{array}$ & 39,00 \\
\hline Custos mais acurados para apreçamento dos produtos ou serviços & 32,00 \\
\hline Melhoria nos processos e práticas de produção e marketing & 25,00 \\
\hline Análise dos custos dos estoques & 7,00 \\
\hline Outros não especificados & 14,00 \\
\hline
\end{tabular}

Como algumas empresas citaram mais de uma característica, a totalização é maior que $100 \%$. Observa-se como a associação do sistema $A B C$ com o planejamento estratégico foi significativa nas respostas. Isso é importante, porque mostra a profundidade com que a empresa conhece o sistema.

\subsection{Grau de acurácia do sistema de custos usado pelas empresas catarinenses}

Com relação à avaliação da acuracidade do sistema de custos usado pelas empresas pesquisadas, a Tabela 4 mostra as respostas apontadas.

TABELA 4 - ACURÁCIA DO SISTEMA DE CUSTOS ADOTADO PELAS EMPRESAS

\begin{tabular}{|lrr|}
\hline Acurácia do sistema de custos & No empresas & \% \\
Muito inacurado & 3 & 4,00 \\
\hline Razoavelmente inacurado & 4 & 5,00 \\
\hline Razoavelmente acurado & 38 & 47,00 \\
\hline Muito acurado & 34 & 42,00 \\
\hline Não disponível & 2 & 2,00 \\
\hline Total & 81 & 100,00 \\
\hline
\end{tabular}


Observa-se que $47 \%$ avaliam o seu sistema de custos como razoavelmente acurado e $42 \%$ das empresas o avaliam como muito acurado, o que vale dizer que $89 \%$ das empresas consideram o seu sistema de custos acurado no sentido de fornecer as informações de custos dos produtos ou serviços.

Quanto à importância do sistema de custos para a tomada de decisão, a Tabela 5 mostra as respostas a esta questão.

\section{TABELA 5 - IMPORTÂNCIA DO SISTEMA DE CUSTOS PARA A TOMADA DE DECISÕES}

\begin{tabular}{|lcc|}
\hline Importância do sistema de custos & №mpresas & $\%$ \\
\hline Muito importante, alta prioridade & 61 & 75,30 \\
\hline Importante, mas não de alta prioridade & 19 & 23,50 \\
\hline Sem importância, raramente usado & 1 & 1,20 \\
\hline Sem importância, nunca usado & 0 & 0,00 \\
\hline Não disponível & 0 & 0,00 \\
\hline Total & 81 & $100 \%$ \\
\hline
\end{tabular}

Nesse universo, $75 \%$ das empresas consideraram muito importante e de alta prioridade o sistema de custos na tomada de decisão; $23 \%$ acham-no importante, mas não altamente prioritário; e apenas $1 \%$ consideram-no sem importância, demonstrando a im- portância que os custos assumem nas suas atividades econômicas.

A velocidade das mudanças na indústria em que as empresas operam é apresentada na Tabela 6, conforme respostas registradas.

\section{TABELA 6 - VELOCIDADE DAS MUDANÇAS NA INDÚSTRIA EM QUE AS EMPRESAS OPERAM}

\begin{tabular}{|lrr|}
\hline Velocidade das mudanças & No empresas & $\%$ \\
Muito rápida & 14 & 17,00 \\
\hline Rápida & 39 & 48,00 \\
\hline Moderada & 24 & 30,00 \\
\hline Lenta & 4 & 5,00 \\
\hline Muito Lenta & 0 & 0,00 \\
\hline Total & 81 & 100,00 \\
\hline
\end{tabular}

A exigência do mercado, no sentido de desenvolver produtos novos para atrair a expectativa do cliente, torna o ciclo de vida dos produtos muito curto, fazendo aumentar a velocidade das mudanças. Notase que $65 \%$ das empresas responderam que essa velocidade é rápida ou muito rápida.
Uma das questões polêmicas no momento, tanto no meio acadêmico quanto nas empresas, sendo também um dos motivos dessa pesquisa, diz respeito ao incremento e controle dos custos indiretos nas empresas. A Tabela 7 mostra como são controlados os custos indiretos das empresas.

\section{TABELA 7 - COMO SÃO CONTROLADOS OS CUSTOS INDIRETOS DAS EMPRESAS}

\begin{tabular}{|lcr|}
\hline Controle dos custos indiretos nas empresas & № empresas & $\%$ \\
Comparação com o orçamento & 33 & 41,00 \\
\hline Comparação com o orçamento flexível & 11 & 13,00 \\
\hline Comparação com o último período & 32 & 40,00 \\
\hline Sem controle & 2 & 2,00 \\
\hline Outro & 3 & 4,00 \\
\hline Total & 81 & 100,00 \\
\hline
\end{tabular}

A grande maioria, $54 \%$ das empresas, fazem o controle dos custos indiretos comparando-os com o orçamento, e $40 \%$ das empresas comparam-nos com o exercício anterior. 


\subsection{Nível de conhecimento sobre o ABC nas empresas de Santa Catarina}

A Tabela 8 mostra o conhecimento do sistema ABC pelas empresas de Santa Catarina que foram pesquisadas.

\section{TABELA 8 - EMPRESAS QUE TÊM CONHECIMENTO DO SISTEMA ABC}

\begin{tabular}{|c|c|c|}
\hline Conhecimento do sistema ABC & № empresas & $\%$ \\
\hline Conhecimento & 75 & 93,00 \\
\hline Não conhecimento & 6 & 7,00 \\
\hline Total & 81 & 100,00 \\
\hline
\end{tabular}

De um universo de 81 empresas pesquisadas, 6 empresas (7\%), das que responderam à pesquisa, informaram que não tinham ouvido falar do sistema ABC. O setor da indústria foi o que apresentou esse percentual de empresas que não tinha ouvido falar do sistema ABC.

\subsection{Motivos que levaram as empresas catarinenses a implantar o ABC}

Os motivos que fizeram com que 28 empresas, das 81 que responderam o questionário, considerassem a implantação do sistema ABC estão indicados na Tabela 9.

\section{TABELA 9 - RAZÕES QUE FIZERAM SUA EMPRESA IMPLANTAR O SISTEMA ABC}

\begin{tabular}{|lcc|}
\hline Razões da implantação do sistema ABC & № Motivos & $\%$ \\
Custos mais acurados para precificação & 9 & 18,00 \\
\hline Análise de lucro mais acurada para tomadas de decisões s/ produtos & 16 & 33,00 \\
\hline Discernimento das causas do aumento ou redução dos custos & 11 & 22,00 \\
\hline Melhoria nos processos e práticas de produção e marketing & 7 & 14,00 \\
\hline Análise dos custos dos estoques & 2 & 4,00 \\
\hline Outros & 4 & 8,00 \\
\hline Total & 49 & 100,00 \\
\hline
\end{tabular}

Ressalte-se que os 49 motivos apresentados pelas empresas são superiores em número às 28 empresas que consideraram alguma vez implantar o sistema $A B C$, em razão de essas empresas apresentarem mais de uma razão.

Predomina o fator "análise do lucro mais acurado para tomada de decisões", escolhido por $33 \%$ das empresas como motivo para usarem o sistema $A B C$; seguido do fator "discernimento das causas do aumento ou a redução de custos" que corresponde a $22 \%$ das empresas.

Curiosamente, os custos mais acurados para a formação dos preços não representam o motivo mais significativo, provavelmente devido ao fato de o mercado ditar os preços.

Por sua vez, o custo para análise dos estoques foi um dos motivos menos importantes apontados pelas empresas pesquisadas (7\%), mesmo porque este serve de base mais para fins fiscais. Isso leva a crer que predomina, nas empresas catarinenses, o interesse pelo uso do custo para fins gerenciais.

A maioria das empresas contratou consultores externos para a elaboração do programa de computação para processar o sistema $A B C$, o que pode ser acompanhado pela Tabela 10. 
TABELA 10 - ELABORAÇÃO DO PROGRAMA DE COMPUTADOR QUE PROCESSA O SISTEMA ABC

\begin{tabular}{|lrr|}
\hline Responsável pela elaboração do programa de computação para o ABC & № Respostas & \% \\
\hline Funcionários da empresa & 2 & 14,00 \\
\hline Consultores externos & 8 & 57,00 \\
\hline Pacote comercial & 4 & 29,00 \\
\hline Total & 14 & 100,00 \\
\hline
\end{tabular}

Em $57 \%$ dos casos, consultores externos elaboraram o programa de computador para processar o sistema ABC; $29 \%$ utilizaram-se de um pacote comercial; e $14 \%$ das empresas desenvolveram o programa pelos funcionários da própria empresa.

Portanto, a elaboração dos programas de computação para processar o sistema $\mathrm{ABC}$, na maioria das empresas pesquisadas, foi por consultores externos à organização, o que pode caracterizar a falta de um conheci- mento mais aprofundado dos funcionários da empresa sobre o funcionamento operacional do sistema ABC.

\subsection{Razão do abandono do ABC após implantado por empresas catarinenses}

A Tabela 11 evidencia as empresas que implantaram ou estão implantando o sistema $A B C$ e as que abandonaram a sua implantação.

\section{TABELA 11 - EMPRESAS QUE ESTÃO IMPLANTANDO OU IMPLANTARAM O SISTEMA ABC}

\begin{tabular}{|lrr|}
\hline Fase de implantação do sistema ABC & № empresas & \% \\
Empresa avaliou e decidiu utilizar & 4 & 33,00 \\
\hline Empresa está implantando & 1 & 8,00 \\
\hline Empresa concluiu sua implantação & 5 & 42,00 \\
\hline Empresa implantou e decidiu abandonar & 2 & 17,00 \\
\hline Total & 12 & 100,00 \\
\hline
\end{tabular}

Observa-se que, entre as 12 empresas das 81 que responderam ao questionário, 4 empresas avaliaram o sistema $A B C$ e decidiram utilizá-lo; 1 empresa está implantando o sistema ABC; 5 empresas já concluíram sua implantação e estão utilizando o sistema $A B C$; e duas empresas já utilizaram o sistema $A B C$ e decidiram abandoná-lo.

As duas empresas que abandonaram o sistema $A B C$, do total de 12 empresas, são do setor têxtil, e as razões do abandono, apontadas pelas empresas, estão relacionadas à complexidade de apropriar os custos e as despesas às atividades e estas aos produtos, à duplicidade de uso dos sistemas, à manutenção onerosa e à dificuldade de separar a produção por unidades de negócios.

\subsection{Ligação do sistema ABC a outros projetos de suporte à gestão das empresas catarinenses}

As respostas à questão que indaga se o sistema $A B C$ está ligado ou não a outros projetos de suporte à gestão empresarial estão registradas na Tabela 12.

\section{TABELA 12-O SISTEMA ABC E SUA LIGAÇÃO COM OUTROS PROJETOS NAS EMPRESAS PESQUISADAS}

\begin{tabular}{|lrr|}
\hline Ligação do sistema ABC a outros projetos da empresa & № empresas & $\%$ \\
\hline Ligado ao sistema “Just-in-Time” & 0 & 0,00 \\
\hline Ligado ao sistema Administração da Qualidade Total (TQM) & 3 & 25,00 \\
\hline Ligado ao sistema Teoria da Restrição & 0 & 0,00 \\
\hline Ligado ao sistema Processo de Reengenharia & 0 & 0,00 \\
\hline Não é ligado a nenhum sistema & 3 & 25,00 \\
\hline Ligado a outro(s) sistema(s) & 6 & 50,00 \\
\hline Total & 12 & 100,00 \\
\hline
\end{tabular}


Nota-se que $50 \%$ das empresas que implantaram o sistema $A B C$ o tem ligado com outros sistemas, $25 \%$ não o tem ligado a nenhum sistema e $25 \%$ o ligaram com o sistema TQM.

\section{COMPARAÇÃO DOS RESULTADOS DA PESQUISA EM EMPRESAS CATARINENSES COM OS ALCANÇADOS NOTRABALHO DE REFERÊNCIA}

A Tabela 13 faz um comparativo entre os resultados dessa pesquisa com os resultados obtidos nas pesquisas realizadas no Brasil (Khoury, 1996), no Canadá (Armitage e Micholson, 1993), na Inglaterra (Innes e Mitchell, 1991) e em duas pesquisas realizadas nos Estados Unidos (Cost Management Update, 1991 e Cost Management Update, 1993).

\section{TABELA 13 - COMPARATIVO DOS RESULTADOS DESSA PESQUISA COM OUTRAS DESSA NATUREZA}

\begin{tabular}{|c|c|c|c|c|c|c|}
\hline $\begin{array}{l}\text { Local onde foi realizada a pesquisa } \\
\text { Ano da pesquisa }\end{array}$ & $\begin{array}{r}\text { Santa } \\
\text { Catarina } \\
2000\end{array}$ & $\begin{array}{r}\text { Brasil } \\
1996\end{array}$ & $\begin{array}{r}\text { Canadá } \\
1992\end{array}$ & $\begin{array}{r}\text { Estados } \\
1991\end{array}$ & $\begin{array}{r}\text { Unidos } \\
1993\end{array}$ & $\begin{array}{r}\text { Inglaterra } \\
1991\end{array}$ \\
\hline Número de empresas pesquisadas & 200 & 500 & 702 & 2500 & 1500 & 720 \\
\hline Números de empresas que responderam à pesquisa & 81 & 283 & 352 & 566 & 400 & 187 \\
\hline Taxa de resposta & $41 \%$ & $57 \%$ & $50 \%$ & $23 \%$ & $27 \%$ & $26 \%$ \\
\hline Empresas que não tinham considerado o sistema ABC & $65 \%$ & $40 \%$ & $67 \%$ & $70 \%$ & $50 \%$ & $52 \%$ \\
\hline Empresas que estavam avaliando o sistema $A B C$ & $6 \%$ & $28 \%$ & $15 \%$ & $19 \%$ & $14 \%$ & $33 \%$ \\
\hline $\begin{array}{l}\text { Empresas que tinham decidido utilizar } \\
\text { o sistema } A B C\end{array}$ & $5 \%$ & $4 \%$ & - & - & - & - \\
\hline $\begin{array}{l}\text { Empresas que tinham avaliado e rejeitaram } \\
\text { o sistema } A B C\end{array}$ & $15 \%$ & $9 \%$ & $4 \%$ & - & - & $8 \%$ \\
\hline $\begin{array}{l}\text { Empresas que tinham implantado ou estavam } \\
\text { implantando o sistema } A B C\end{array}$ & $7 \%$ & $18 \%$ & $14 \%$ & $11 \%$ & $36 \%$ & $6 \%$ \\
\hline Empresas que abandonaram o sistema $A B C$ & $2 \%$ & $1 \%$ & - & - & 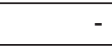 & $1 \%$ \\
\hline Total & $100 \%$ & $100 \%$ & $100 \%$ & $100 \%$ & $100 \%$ & $100 \%$ \\
\hline
\end{tabular}

Fonte: KHOURY, Carlos Yorghi. ABC - Sistema de custos baseado em atividades: uma pesquisa de sua utilização no Brasil. Tese de Doutorado - Fundação Getúlio Vargas, Escola de Administração de Empresas de São Paulo. São Paulo, 1997.

Das empresas pesquisadas em Santa Catarina, $65 \%$ não se interessaram em considerar a implantação do sistema ABC. Este percentual é superior aos $40 \%$ constatados nas 500 maiores empresas pesquisadas no Brasil. Porém, é menor do que os percentuais apurados nas pesquisas realizadas no
Canadá (67\%) e em uma dos Estados Unidos (70\%).

O percentual de empresas que tinham avaliado e depois rejeitaram, bem como o percentual de empresas que abandonaram o sistema ABC, de acordo com as pesquisas realizadas em Santa Catarina e no Brasil, está retratado na Tabela 14.

TABELA 14 - EMPRESAS QUE REJEITARAM E EMPRESAS QUE ABANDONARAM O SISTEMA ABC

\begin{tabular}{|lcc|}
\hline & Pesquisa em & Pesquisa \\
Rejeição e abandono do sistema ABC & Santa Catarina & no Brasil \\
Avaliaram e rejeitaram o sistema ABC & $15 \%$ & $9 \%$ \\
\hline Abandonaram o sistema ABC & $2 \%$ & $1 \%$ \\
\hline
\end{tabular}


Observa-se que, em Santa Catarina, $15 \%$ das empresas avaliaram e rejeitaram o sistema $A B C$, e no Brasil $9 \%$. Por outro lado, $2 \%$ das empresas de Santa Catarina que responderam o questionário abandonaram o sistema ABC. No Brasil esse percentual foi de $1 \%$.

A Tabela 15 mostra se o sistema ABC, em Santa Catarina, bem como no Brasil, suplementou ou substituiu o sistema tradicional de custos.

TABELA 15 - O SISTEMA ABC SUPLEMENTOU OU SUBSTITUIU O SISTEMA DE CUSTOS TRADICIONAL

\begin{tabular}{|c|c|c|}
\hline Suplementação ou substituição do sistema ABC & $\begin{array}{l}\text { Pesquisa em } \\
\text { Santa Catarina }\end{array}$ & $\begin{array}{l}\text { Pesquisa } \\
\text { no Brasil }\end{array}$ \\
\hline Suplementou & $8 \%$ & $64 \%$ \\
\hline Substituiu & $42 \%$ & $14 \%$ \\
\hline Suplementou ou substituiu em algumas partes & $33 \%$ & $20 \%$ \\
\hline Será decidido futuramente & $17 \%$ & $2 \%$ \\
\hline
\end{tabular}

Na comparação das duas pesquisas, observa-se que é elevado o percentual, $42 \%$, das empresas de Santa Catarina que decidiram pela substituição do sistema tradicional de custos. No Brasil, o percentual foi bem menor, $14 \%$. No quesito "suplementar as informações", o resultado de Santa Catarina foi baixo,
$8 \%$ das empresas pesquisadas, ao contrário da pesquisa ocorrida no Brasil, em que o percentual para suplementar as informações foi de $64 \%$.

Com relação à integração do sistema $A B C$ com outros sistemas, os números da pesquisa são evidenciados na Tabela 16.

\section{TABELA 16 - INTEGRAÇÃO DO SISTEMA ABC COM OUTROS SISTEMAS}

\begin{tabular}{|c|c|c|}
\hline Integração do sistema $A B C$ & $\begin{array}{l}\text { Pesquisa em } \\
\text { Santa Catarina }\end{array}$ & $\begin{array}{l}\text { Pesquisa } \\
\text { no Brasil }\end{array}$ \\
\hline Sistema independente & $25 \%$ & $30 \%$ \\
\hline Integrado com sistema contábeis & $50 \%$ & $38 \%$ \\
\hline Integrado com outros sistemas & $33 \%$ & $48 \%$ \\
\hline Sem resposta & $0 \%$ & $2 \%$ \\
\hline
\end{tabular}

Verifica-se nas duas pesquisas um percentual semelhante: nas empresas de Santa Catarina, 25\% dos respondentes, enquanto na pesquisa realizada no Brasil, $30 \%$ decidiram pelo sistema de custos independente.

Observa-se na Tabela 17 as diferenças de preferência entre as empresas com relação à elaboração do programa de computador do sistema ABC.

TABELA 17 - ELABORAÇÃO DO PROGRAMA DE COMPUTADOR QUE PROCESSA O SISTEMA ABC

\begin{tabular}{|c|c|c|}
\hline $\begin{array}{l}\text { Responsável pela elaboração do programa de } \\
\text { computador para o sistema ABC }\end{array}$ & $\begin{array}{l}\text { Pesquisa em } \\
\text { Santa Catarina }\end{array}$ & $\begin{array}{l}\text { Pesquisa } \\
\text { no Brasil }\end{array}$ \\
\hline Funcionários da empresa & $17 \%$ & $50 \%$ \\
\hline Consultores externos & $67 \%$ & $44 \%$ \\
\hline Pacote comercial & $33 \%$ & $34 \%$ \\
\hline
\end{tabular}

As empresas de Santa Catarina usaram menos os funcionários da empresa, $17 \%$, e muito mais os consultores externos, $67 \%$. Ao contrário dos percentuais das empresas pesquisadas no Brasil, onde $50 \%$ das empresas utilizaram seus funcionários para desenvolver o sistema e $44 \%$ utilizaram-se de consultores externos.

A Tabela 18 mostra os dados comparativos de Santa Catarina e do Brasil sobre a acurácia do sistema de custos usado pelas empresas. 
TABELA 18 - AVALIAÇÃO DA ACURÁCIA DO SISTEMA DE CUSTOS EMPREGADO PELAS EMPRESAS

\begin{tabular}{|lrr|}
\hline Acurácia do sistema de custos empregado & $\begin{array}{r}\text { Pesquisa em } \\
\text { Santa Catarina }\end{array}$ & $\begin{array}{r}\text { Pesquisa } \\
\text { no Brasil }\end{array}$ \\
Muito inacurado & $4 \%$ & $2 \%$ \\
\hline Razoavelmente inacurado & $5 \%$ & $0 \%$ \\
\hline Razoavelmente acurado & $47 \%$ & $54 \%$ \\
\hline Muito acurado & $42 \%$ & $44 \%$ \\
\hline Informações de Custos não Disponíveis & $2 \%$ & $0 \%$ \\
\hline
\end{tabular}

Verifica-se que os números ficaram próximos nas empresas pesquisadas em Santa Catarina e nas pesquisadas no Brasil, no que concerne a avaliação da acurácia do seu sistema de custos.

\section{CONCLUSÕES DA PESQUISA}

A presente pesquisa teve como objetivo verificar como está sendo utilizado o sistema de custeio baseado em atividades - ABC (Activity Based Costing) no processo de gestão empresarial nas maiores empresas de Santa Catarina, o que constitui uma réplica da pesquisa realizada nas maiores empresas no Brasil, por Carlos Yorghi Khoury, além de verificar até que ponto elas estão relacionadas.

Os dados da pesquisa foram obtidos através de questionário enviado para cada uma das 200 maiores empresas privadas catarinenses, com retorno de 81 questionários respondidos, ou seja, representando $41 \%$ das empresas, uma taxa de retorno que pode ser considerada significativa.

A pesquisa realizada, através da análise dos dados coletados nestas empresas, procurou enfocar os aspectos que seguem:

\section{a) Avaliação do grau de acuracidade do sistema de custos usado na empresa}

Um percentual pequeno, apenas $4 \%$, considera muito inacurado o sistema de custos de sua empresa. Por outro lado, um número significativo de empresas (89\%) respondeu que avalia como "razoavelmente acurado e muito acurado". Nota-se que o sistema de custos vem sendo muito utilizado, pelas empresas catarinenses, como um instrumento administrativo imprescindível para a análise e controle de seus negócios. A grande maioria, $75 \%$ das empresas pesquisadas, tam- bém considera muito importante e de alta prioridade o sistema de custos como suporte à tomada de decisão na sua empresa, sendo que apenas $1 \%$ o considera sem importância e, portanto, raramente usado na empresa.

b) Nível de conhecimento do sistema $A B C$ na empresa

Verificou-se que 75 das empresas que responderam à pesquisa, ou seja, 93\%, tinham conhecimento do sistema $A B C$, o que vale dizer que um percentual pequeno, de apenas $7 \%$, não tinha conhecimento do sistema. Portanto, há um alto índice de conhecimento e de informação sobre o sistema $A B C$, revelando que o mesmo teve boa propagação entre as empresas localizadas em Santa Catarina.

\section{c) Grau de interesse em implantar o sistema $A B C$} na empresa

Das 81 empresas que responderam ao questionário, 28 empresas consideraram alguma vez implantar o sistema $\mathrm{ABC}$, correspondendo a $35 \%$ da amostra. Dessas, 18\% estão atualmente ainda avaliando o sistema $A B C$, representando futuros potenciais usuários do sistema. Um percentual significativo, 39\% das empresas, avaliou e decidiu não utilizar o sistema $A B C$.

Os motivos mais fortes da rejeição na implantação do sistema $A B C$, apontados pelas empresas, foram: $30 \%$ responderam que o sistema que vem sendo usado atende às necessidades da empresa, o que revela que a contabilidade de custos tradicional é útil na definição da estratégia empresarial. Por sua vez, $19 \%$ rejeitaram o mesmo devido à complexidade da implantação e operação do sistema; $11 \%$ afirmaram que o sistema 
ABC não é adequado para o seu negócio.

Por outro lado, 12 empresas, em Santa Catarina, representando $44 \%$ das que mostraram interesse no sistema, estão implantando ou estão utilizando o sistema ABC. Interessante observar que são todas do setor industrial.

\section{d) Motivos que levaram a empresa implantar o sistema $A B C$}

A maior competição que enfrentam, a alta diversificação de seus produtos e a elevada velocidade de mudanças em que operaram são os principais motivos que puderam ser detectados pelo interesse das empresas catarinenses na implantação do sistema ABC.

As principais razões que fizeram as empresas considerarem a sua implantação foram: $33 \%$ das empresas - análise do lucro mais acurado para tomada de decisão sobre que produtos produzir ou que serviços prestar; $22 \%$ das empresas discernimento das causas do aumento ou redução dos custos; $18 \%$ das empresas - os custos mais acurados para a precificação dos produtos. Estes foram os principais aspectos favoráveis apontados pelas empresas para a utilização do sistema ABC. A análise dos custos dos estoques (7\%) foi apontada como de menor importância, mesmo este servindo de base fiscal compulsória para as empresas.

\section{e) Razões do abandono do sistema ABC após sua implantação}

Duas empresas, das 12 que estavam implantando ou estavam utilizando, decidiram abandonar o sistema $A B C$. As razões do abandono apontadas por essas empresas foram: a complexidade de apropriar os custos e despesas das atividades aos produtos, a duplicidade de uso dos sistemas, a manutenção onerosa e a dificuldade de separar os custos na produção por unidade de negócio. Apesar de restringir-se a duas empresas, isso leva a crer que o sistema $A B C$ não pode ser visto como um sistema que está favorecendo a todas as empresas que o estão implantando, como se fosse uma solução para todos os males.

A complexidade da implantação e operação do sistema, a dificuldade de manutenção, além do alto custo para a sua implantação e a subjetividade das informações sobre a distribuição dos custos indiretos foram as razões pelas quais as empresas decidiram não utilizar o sistema ABC.

\section{f) Outros métodos de suporte que estão sendo usados na gestão das empresas}

Observou-se que, em $25 \%$ das empresas, o sistema $A B C$ está ligado ao Sistema de Administração da Qualidade Total (TQM); em 50\%, ligado a outros sistemas não identificados e, em $25 \%$ das empresas, o sistema $A B C$ não está ligado a nenhum sistema.

Por outro lado, $46 \%$ das empresas informaram que o sistema $A B C$ está integrado com sistemas contábeis.

Importante registrar, também, que 50\% das empresas tiveram o patrocínio e apoio do presidente da empresa. Não obstante, os programas de computação foram desenvolvidos, em $57 \%$ das empresas, por consultores externos.

\section{g) Semelhanças e diferenças no resultado da pesquisa das 200 maiores empresas de Santa Catarina com a pesquisa feita nas 500 maio- res empresas do Brasil}

Foram efetuadas diversas comparações entre as pesquisas realizadas em Santa Catarina e nas 500 maiores empresas do Brasil. Destacam-se, primeiramente, as principais semelhanças encontradas: $\checkmark$ na avaliação quanto à acurácia do sistema de custos utilizado pelas empresas - em Santa Catarina, $42 \%$ dos entrevistados consideram seu sistema acurado; no Brasil, de modo semelhante, $44 \%$;

$\sqrt{ }$ na elaboração do programa de computação para processar o sistema ABC - em Santa Catarina 33\% das empresas pesquisadas usaram pacote comercial; no Brasil, um percentual muito próximo, $34 \%$; e

$\checkmark$ duas empresas (2\% das pesquisadas) de Santa Catarina e duas empresas ( $1 \%$ das pesquisadas) no Brasil implantaram e resolveram abandonar o sistema $A B C$.

No que concerne às diferenças encontradas nas duas pesquisas realizadas, cabe ressaltar os seguintes aspectos: 
$\sqrt{ }$ as empresas que não tinham interesse na implantação do sistema ABC, em Santa Catarina totalizaram 65\%; e, no Brasil, $40 \%$;

$\sqrt{ }$ o sistema ABC suplementou o sistema de custos tradicional em $8 \%$ das empresas pesquisadas em Santa Catarina, percentual bem inferior aos 64\% das empresas do Brasil;

$\sqrt{ }$ o percentual de empresas que substituiu o sistema $\mathrm{ABC}$, em Santa Catarina representou $42 \%$; e, no Brasil, $14 \%$;

$\sqrt{ }$ as empresas que avaliaram e rejeitaram o sistema $A B C$, representou $15 \%$ nas empresas de Santa Catarina, e $9 \%$ nas empresas do Brasil.

\begin{abstract}
Deve-se também observar que a pesquisa em Santa Catarina foi realizada 4 anos após a realizada no Brasil, o que vale dizer que o resultado da pesquisa realizada no Brasil agora poderia ser outro. Tal suspeita decorre do fato de um expressivo percentual, $58 \%$, das empresas de Santa Catarina ter implantado este sistema recentemente, ou seja, a menos de dois anos. A pesquisa mostrou, como referência para a reflexão, que o sistema $A B C$ não está sendo o remédio para a cura de todos os males, como se estava propagando, e a sua utilização não está sendo feita pelo número desejado de empresas e na velocidade esperada.
\end{abstract}

\title{
REFERÊNCIAS BIBLIOGRÁFICAS
}

BRIMSON, James A. Contabilidade por atividades: uma abordagem de custeio baseado em atividades. São Paulo: Atlas, 1996.

CHALOS, Peter. Managing cost in today's manufacturing environment. New Jersey: Prentice-Hall, 1992.

CHING, Hong Yuh. Gestão baseada em custeio por atividades = ABM - Activity Based Management. São Paulo: Atlas, 1995

GOGAN, Samuel. Activity based costing (ABC): a poderosa estratégia empresarial. 2 ed. São Paulo: Pioneira, 1994.

KAPLAN, Roberto S. e COOPER, Robin. Custo \& desempenho: administre seus custos para ser mais competitivo. São Paulo: Futura, 1998.

KHOURY, Carlos Yorghi. ABC - sistema de custos baseado em atividades: uma pesquisa de sua utilização no Brasil. Tese de Doutorado - Fundação Getúlio Vargas, Escola de Administração de Empresas de São Paulo. São Paulo, 1997.

KHOURY, Carlos Yorghi. ABC - sistema de custos baseado em atividades: uma pesquisa de sua utilização no Brasil. RAE - Revista de Administração de Empresas. São Paulo, FGV. v. 39, n. 4, p. 55-63, out./dez. 1999.

MARTINS Eliseu. Contabilidade de custos. 2 ed. São PauIo: Atlas, 1984.

NAKAGAWA, Masayuki. ABC - Custeio Baseado em Atividade. São Paulo: Atlas, 1994.

SAKURAI, Michiharu. Gerenciamento integrado de custos. São Paulo: Atlas, 1997.

SHANK, John K. e GOVINDARAJAN, Viajay. A revolução dos custos: como reinventar e redefinir sua estratégia de custos para vencer em mercados crescentes competitivos. 2 ed. Rio de Janeiro: Campus, 1997. 